\title{
The impact of the climate crisis on brain health
}

\author{
Long-term exposure to air pollution is harmful to human health, causing damage to respiratory and \\ cardiovascular systems. A new study provides evidence that even short-term, relatively low-level air pollution \\ can be detrimental for cognitive function, and suggests the possibility that a commonly used drug might help \\ reduce the harmful effects.
}

\section{Joanne Ryan and Alice J. Owen}

\begin{abstract}
n 2020, the COVID-19 pandemic wreaked havoc worldwide. However, as 'non-essential' industries ground to a halt, flights were cancelled and many people stayed at home, this provided a rare glimpse into the benefits of a reduction in pollution-generating human activities. The air got a little bit cleaner. Satellite data showed that there was a reduction in air pollution measured from air quality stations across 34 countries $^{1}$. The average $31 \%$ drop in fine particulate matter $\left(\mathrm{PM}_{2.5}\right)$ was a brief reprieve not just for our planet, but also our health. A study published in this issue of Nature Aging by Gao et al. indicates that short-term exposure to $\mathrm{PM}_{2.5}$, even at relatively low levels, is damaging to brain health².
\end{abstract}

$\mathrm{PM}_{2.5}$ are particles smaller than $2.5 \mathrm{~mm}$. They are so small that they can penetrate deep into the lungs and circulatory system, causing adverse health effects. The major source of $\mathrm{PM}_{2.5}$ throughout the world is from the burning of fossil fuels; in particular, for transportation, industry and generating electricity, as well as agriculture. $\mathrm{PM}_{2.5}$ has been ranked as the thirteenth leading cause of worldwide mortality, contributing more than 800,000 premature deaths each year ${ }^{3}$. Indeed, air pollution is a major public health problem around the world, being the second leading cause of death from non-communicable diseases. Air pollution has well-established negative effects on respiratory and cardiovascular health, including acute difficulty with breathing, coughing and chest tightness, and longer-term impacts on cardiovascular morbidity and mortality ${ }^{4}$. Increasingly, pollution is recognized for its detrimental effects on a range of other systems and organs, too.

The study by Gao et al. included participants from the Normative Aging Study, which has followed adult men from the Veterans Affairs Outpatient Clinic in Boston, Massachusetts, regularly since 1963. The authors looked at data from almost

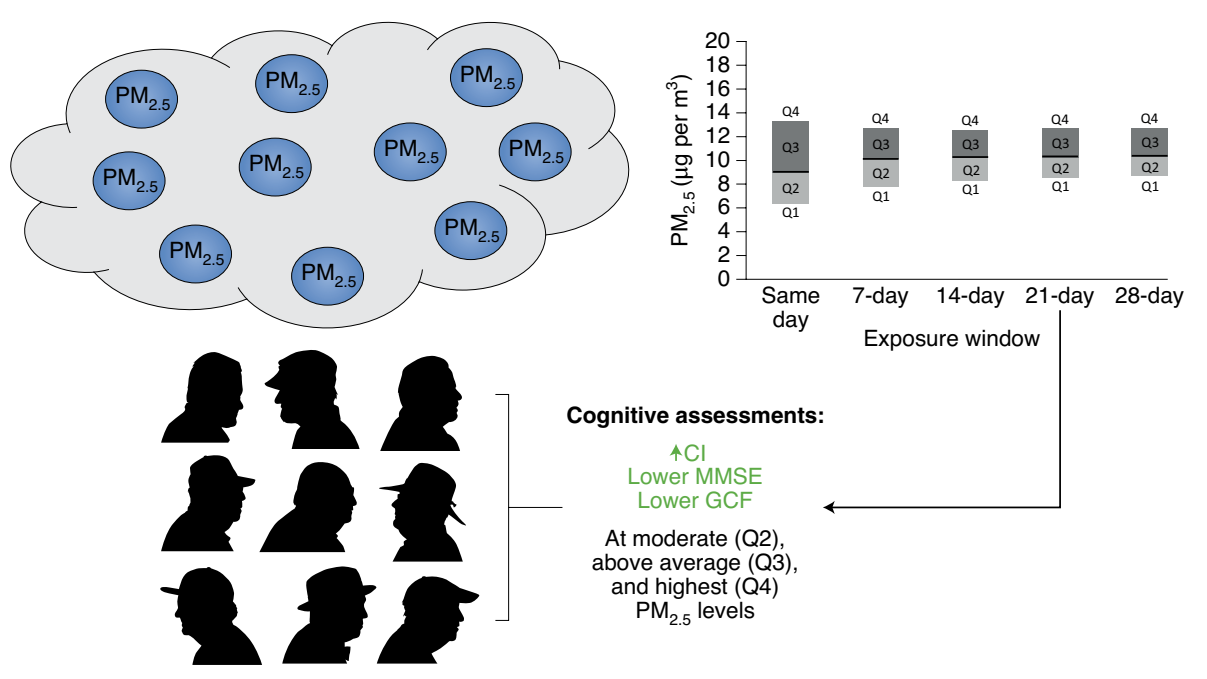

Fig. 1 | Short-term air pollution and cognitive function. The upper graph shows the levels of $\mathrm{PM}_{2.5}$ exposure measured over five different time periods, from the lowest (Q1) to highest (Q4) quartile; the black lines represent the median level over the time period. Among a cohort of older males, exposure to even moderate (Q2) levels of $\mathrm{PM}_{2.5}$ over a 21-day period was associated with an increased risk of cognitive impairment $(\mathrm{Cl})$, as well as lower MMSE and global composite function (GCF) scores. Higher exposure levels further increased risk. Silhouette icons: RobinOlimb / DigitalVision Vectors / Getty.

1,000 older men, with an average age of 69 years, during the period of 1995 to 2012 . These men had participated in repeated cognitive assessments to test their attention, learning and memory (which the authors combined to create a global cognitive function score), and in the Mini Mental State Examination (MMSE), which helps detect early signs of dementia. Air pollution particles, $\mathrm{PM}_{2.5}$ and black carbon, which is a tracer of vehicle traffic and a key component of $\mathrm{PM}_{2.5}$, were recorded on the day of the cognitive assessments. The average levels at 7, 14, 21 and 28 days prior to each cognitive-testing visit were also determined.

The authors then compared men's cognitive performance according to their air pollution exposure, and the findings were quite startling. Even relatively small increases in the levels of $\mathrm{PM}_{2.5}$ in the 3-4 weeks prior to testing were associated with consistently worse cognitive performance (Fig. 1). More specifically, an average 21-day exposure of $\mathrm{PM}_{2.5}$ between 8.52 to 10.25 $\mu \mathrm{g}$ per $\mathrm{m}^{3}$, compared to less than $8.52 \mu \mathrm{g}$ per $\mathrm{m}^{3}$, was associated with lower global cognitive function and MMSE score, and an increased risk of low MMSE, indicating possible dementia. Levels higher than this further increased risk, but in a non-linear fashion. Furthermore, higher pollution over a very short period (7-day average over $10.13 \mu \mathrm{g}$ per $\mathrm{m}^{3}$ ) resulted in worse MMSE scores and a higher risk of having low cognition. The findings with black carbon were not as strong but followed a similar pattern. Boston, the city in which this study took place, has by no means the worst air quality in the USA or the world, and yet significant detrimental effects of air pollution on cognitive function were observed. To put their values into context, 
global $\mathrm{PM}_{2.5}$ levels were around $44 \mu \mathrm{g}$ per $\mathrm{m}^{3}$ in 2015 (ref. ${ }^{5}$ ), and the WHO air quality guidelines target is $10 \mu \mathrm{g}$ per $\mathrm{m}^{3}$.

Evidence is increasing that air pollution, including $\mathrm{PM}_{2.5}$, could be a risk factor for dementia ${ }^{6}$. High levels of $\mathrm{PM}_{2.5}$ have also been associated with cognitive decline and smaller brain volume ${ }^{7}$. Most previous studies, however, only considered long-term exposure and generally high levels of air pollution. They also focused predominantly on dementia, which is a severe impairment of cognition that affects daily functioning. It remained unclear to what extent pollution could also impact general cognition. The results of the current study are especially important because they provide some of the first evidence that even relatively low-level, short-term increases in $\mathrm{PM}_{2.5}$ are detrimental for thinking and memory, as well as global cognition in older adults.

Further strengthening their results, the authors controlled for a range of other factors which could have impacted their findings, including accounting for temporal trends, seasonal differences and meteorological conditions (temperature and humidity levels), which all affect the levels of air pollution. A limitation of their work is that they used ambient air pollution levels at central sites rather than personal monitoring of exposure. But the latter is logistically difficult to achieve in a large cohort study, and prior data from the Boston area indicate a good correlation between the two measures ${ }^{8}$. While findings from the current study were specific to white male veterans with an average age of 73 years, and thus an older population who is more susceptible to cognitive decline, they are likely to be generalizable to the larger population. For example, exposure to high levels of air pollution has been shown to affect brain development in children ${ }^{9}$, and women appear even more susceptible to the effects of air pollution on respiratory health than men ${ }^{10}$. Evidence that there is widening inequality in air pollution exposure ${ }^{11}$ highlights the need to better understand how strategies to reduce $\mathrm{PM}_{2.5}$ exposure can be effectively implemented for everyone.

The way in which air pollution negatively impacts health appears to involve, in part, activation of the body's inflammatory response $^{12}$. An additional aspect of the study by Gao et al. was to determine whether the effects they observed in their study were offset by the use of commonly prescribed anti-inflammatory medications. They found that men using non-steroidal anti-inflammatory drugs (NSAIDs), such as aspirin, appeared somewhat protected, with a diminished association between air pollution and cognition. In contrast, moderate and high levels of air pollution were associated with a stronger negative effect in men not using NSAIDs. These findings are exciting. They align with the supposed causal mechanisms underpinning the negative effects of $\mathrm{PM}_{2.5}$ on human health and support the role of inflammation in cognitive decline and dementia ${ }^{13}$. Although it is unclear whether NSAIDs can directly reduce cognitive impairment ${ }^{14}$, and no direct association was observed in Gao's study, it is plausible that NSAIDs could attenuate the inflammation induced by $\mathrm{PM}_{2.5}$. Another possible explanation for the findings is that NSAIDs might help reduce the alterations in cerebrovascular hemodynamics that can occur with $\mathrm{PM}_{2.5}$ exposure ${ }^{15}$; thus, in turn, minimizing vascular-related cognitive impairment.

However, at this early stage we need to be very cautious in not over-interpreting the data. There are likely to be many other differences between men who use NSAIDs and those who don't, and observational studies like the one by Gao et al. can't fully tease these apart. For example, the use of NSAIDs might be associated with better access to healthcare or health literacy, and these factors could not be explored in Gao's analysis. The findings provide a tantalizing possibility of better understanding the role of air pollution on cognitive health, and more research into the underlying mechanisms of NSAIDs' potential protective effects is urgently needed.

The study by Gao and colleagues is an important piece of work that highlights the effect that environmental pollution is having on our health, with even short-term increases in $\mathrm{PM}_{2.5}$ exposure being a major threat to cognitive function. These results are important because the implications for public health, and consequent health, societal and economic costs of air pollution, are immense. This should be a further wake-up call about the urgent need for action. It has been estimated that $90 \%$ of the world's population breathe polluted air: air that fails to meet the limits as set out in the WHO air quality guidelines ${ }^{3}$. Breathing clean air is fundamental to our health but represents a global challenge and one of substantial inequity, disproportionally affecting the most vulnerable ${ }^{11}$. Even in places where air pollutant emissions have been regulated by governments and agencies for decades and are decreasing, short-term spikes in air pollution remain frequent. Furthermore, many other locations are experiencing worsening air quality. Air pollution is not only damaging to the planet but also to our health, including being detrimental to thinking and memory, and quite possibly representing a contributing factor to rising dementia incidence.

\section{Joanne Ryan (iD) and Alice J. Owen} School of Public Health and Preventive Medicine, Monash University, Melbourne, Victoria, Australia.

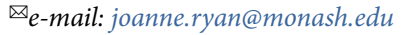

Published online: 3 May 2021 https://doi.org/10.1038/s43587-021-00062-2

References

1. Venter, Z. S., Aunan, K., Chowdhury, S. \& Lelieveld, J. Proc. Natl Acad. Sci. USA 117, 18984-18990 (2020).

2. Gao, X. et al. Nat. Aging https://doi.org/10.1038/s43587-02100060-4 (2021).

3. Ambient Air Pollution: a Global Assessment of Exposure and Burden of Disease (WHO, 2016).

4. Newby, D. E. et al. Eur. Heart. J. 36, 83-93 (2015).

5. Cohen, A. J. et al. Lancet 389, 1907-1918 (2017).

6. Peters, R. et al. J. Alzheimers Dis. 70, S145-S163 (2019).

7. Power, M. C., Adar, S. D., Yanosky, J. D. \& Weuve, J. Neurotoxicology 56, 235-253 (2016).

8. Sarnat, J. A., Brown, K. W., Schwartz, J., Coull, B. A. \& Koutrakis, P. Epidemiology 16, 385-395 (2005).

9. Rees, N. Danger in the Air: How Air Pollution Can Affect Brain Development in Young Children (UNICEF, 2017).

10. Clougherty, J. E. Environ. Health Perspect. 118, 167-176 (2010).

11. Landrigan, P. J. et al. Lancet 391, 462-512 (2018).

12. Pope, C. A. III et al. Circulation 109, 71-77 (2004).

13. Calderón-Garcidueñas, L. et al. Toxicol. Pathol. 32, 650-658 (2004).

14. Ryan, J. et al. Neurology 95, e320-e331 (2020).

15. Wellenius, G. A. et al. Stroke 44, 1532-1536 (2013).

Competing interests

The authors declare no competing interests. 